Research Article

\title{
Adaptive Boundary Control of Flexible Manipulators with Parameter Uncertainty Based on RBF Neural Network
}

\author{
Cang He, ${ }^{1,2}$ Fang Zhang $\mathbb{D}^{1,2}$ and Jinhui Jiang ${ }^{1,2}$ \\ ${ }^{1}$ State Key Lab of Mechanics and Control of Mechanical Structures, Nanjing University of Aeronautics and Astronautics, \\ 29 Yudao Street, Nanjing 210016, China \\ ${ }^{2}$ College of Aerospace Engineering, Nanjing University of Aeronautics and Astronautics, 29 Yudao Street, Nanjing 210016, China
}

Correspondence should be addressed to Fang Zhang; zhangfangyy@163.com

Received 20 December 2019; Revised 18 September 2020; Accepted 27 September 2020; Published 16 November 2020

Academic Editor: Fabio Rizzo

Copyright $\odot 2020$ Cang He et al. This is an open access article distributed under the Creative Commons Attribution License, which permits unrestricted use, distribution, and reproduction in any medium, provided the original work is properly cited.

\begin{abstract}
In this paper, nonlinear dynamical equations of the flexible manipulator with a lumped payload at the free end are derived from Hamilton's principle. The obtained model consists of both distributed parameters and lumped parameters, namely, partial differential equations (PDEs) governing the flexible motion of links and boundary conditions in the form of ordinary differential equations (ODEs). Considering the great nonlinear approximation ability of the radial basis function (RBF) neural network, we propose a combined control algorithm that includes two parts: one is a boundary controller to track the desired joint positions and suppress the vibration of flexible links; another is a RBF neural network designed to compensate for the parametric uncertainties. The iteration criterion of the RBF neural network weight matrix is derived from the extended Lyapunov function. Stabilization analysis is further carried out theoretically via LaSalle's invariance principle. Finally, the results of the numerical simulation verify that the proposed control law can realize the asymptotic convergence of tracking error and suppression of the elastic vibration as well.
\end{abstract}

\section{Introduction}

Flexible manipulators are widely used in automation field including space station solar array and advanced robots, as well as mechanical manufacture. In contrast with their rigid counterparts, flexible manipulators own several advantages, such as smaller size, lower energy consumption, more workspace, lighter weight, and more portability. However, since the flexible manipulator is a large-span structure with great elasticity and geometrical nonlinearity, the vibration of the link induced during the motion is inevitable [1]. In addition, the parameters of the system are generally partially known and time varying. Therefore, it is hard to obtain the accurate mathematical model of flexible manipulators, which could further cause the control strategies to become even more complex. The dynamics of such class of systems is usually governed by a combination of ODEs, PDEs, and a set of boundary conditions. Conventional control algorithms for parameter uncertainty are generally constructed on dynamic models derived from the assumed mode method or finite element method [2-4], both using mode truncations. In spite of the convenience, some defects still exist in these methods, such as spillover instability caused by neglected higher modes, error of mode truncations, and higher order of the controller. To describe the elastic vibration of the flexible manipulator system more completely, it is rather suitable to establish a series of PDE equations to describe the dynamics of the system.

In view of the aforementioned problems, it is quite a challenging task to design a controller directly without knowing the system dynamics completely. Furthermore, due to the payload variation, the exact values of the payload mass or inertial during the operation are generally unknown. Boundary control is a feedforward control method based on PDE dynamic models and derives from ODE boundary conditions, which has been developed to obtain ideal control results for these PDE model-based systems. In this control method, both joint motor and force actuator are used as 
control inputs. Nguyen et al. used Lyapunov theory to design an exponentially stable boundary controller for the robot arm, which could avoid the spillover problem [5]. Liu et al. established a boundary control algorithm for a flexible double-link manipulator based on the PDE dynamic model [6]. Based on the infinite-dimensional dynamic model, He et al. utilized an output feedback method to represent a boundary controller with input backlash [7]. Jiang et al. established a boundary control algorithm for an output constrained flexible double-link manipulator and proved by a barrier Lyapunov function [8]. However, this study ignored the uncertainty of the joint mass, which also has much influence on the dynamics of flexible manipulator. In such cases, artificial neural networks (ANNs) are suitable to be used as an adaptive controller since it requires relatively less information of the system dynamics. According to its different mechanisms in control field, ANN can be divided into two categories: one is used to establish the model of the dynamic system [9-11], especially for nonlinear system [12]; another is efficient in predicting the dynamic behavior of the partially defined dynamic system with uncertain parameters [13]. Thus, many researchers focused on using ANN to design an adaptive controller for flexible manipulators in order to track the trajectory error of the payload $[14,15]$. Besides, other cooperative control strategies interacted with ANN were proposed for control problems of flexible manipulators in previous studies [16], including ANN controllers combined with PID control law $[17,18]$ or sliding mode control law $[19,20]$.

Recently, RBF neural network (NN) applied in control filed has attracted more and more research interest. RBF $\mathrm{NN}$ was first proposed by Broomhead and Lowe who used it in the prediction of the chaotic time series [21]. Due to the great global approximation capability and learning ability of RBF NNs, many researchers studied its application in approximating the inverse dynamics of flexible manipulators [22]. Moreover, Rogers et al. applied RBF $\mathrm{NN}$ to generate an orthogonal basis to estimate the uncertain parameters [23]. He et al. proposed one RBF NN to approximate the estimated input dead-zone effect, and another RBF $\mathrm{NN}$ is used to estimate the unknown dynamics of the flexible manipulator [24]. However, two RBF NNs for different uncertain parameters, respectively, will increase the complexity of the control system. The synchronization between different networks also needs to be carefully considered. Therefore, using one RBF NN to compensate for all uncertain parameters provides a more efficient and concise way to control the flexible manipulator. To the best of the authors' knowledge, none of the previous researchers have ever used RBF NN to build a combined controller with the boundary control method. In the present study, we first use RBF NN to compensate for the multi-parameter uncertainty, further realizing online learning during the manipulator operation. The uncertain function constructed in our work is time varying, which consists of two different parameters. In comparison with previous work, we note that the outlined novelty of this paper
(1) Utilizes a more complete dynamic model containing multiuncertain parameters and build the co-influence function of the uncertainty

(2) Combines the boundary control with RBF NN to deal with different parameter uncertainties

In this paper, Hamilton's principle is used to derive the dynamic equations of a two-link flexible manipulator. The second joint mass and payload mass are both uncertain in the dynamic model. Then, the dynamic model is simplified under some reasonable assumptions. A combined control law including boundary control algorithm and adaptive RBF NN control algorithm is proposed to track the angular positions and suppress the vibration of the flexible links, respectively.

The rest of this paper is organized as follows. Dynamic equations of the flexible manipulator are derived in Section 2. Adaptive combined control law consisting of boundary control strategy and RBF NN is proposed in Section 3. Stability analysis and Lyapunov function of the control strategy are established in Section 4. Simulation study is carried out in Section 5 and some conclusions are given in the end.

\section{Dynamic Model}

Consider a two-link flexible manipulator holding a payload at its free end in a horizontal plane illustrated in Figure 1. $O_{1} X_{1} Y_{1}$ is the global inertial coordinate system. $O_{1} x_{1} y_{1}$ and $\mathrm{O}_{2} x_{2} y_{2}$ are body-fixed coordinate systems fixed on the first and second link, respectively. We assume that the two links are both modelled as Euler-Bernoulli beams, flexible only in a direction transverse to their principal longitudinal axes in the plane of motion. Consequently, neither out-of-plane deflections nor axial elongation exist in this system. The payload is a rigid body with mass $m_{t}$. Link $i(i=1,2)$ has length $L_{i}$ and moment of inertia $J_{i} . \rho_{i}$ and $E I_{i}$ represent uniform linear density and uniform flexural rigidity of the flexible link $i$, respectively. $J_{t i}$ denotes the moment of inertia of the $i$ th link tip, where $J_{t 2}$ includes the moment of inertia of the payload. Motor $i$ located on the joint $O_{i}$ has lumped mass $m_{i}$ and moment of inertia $J_{m i}$, where $m_{2}$ contains the mass of the tip of link 1. $\tau_{i}$ represents the input torque generated by motor $i$, while $f_{i}$ is the input force generated by the actuator at the tip of link $i$. $\mathbf{r}_{\mathbf{i}}$ stands for the absolute position vector of each point of link $i$ in the global coordinate system. $u_{i}\left(x_{i}, t\right)$ is the elastic deformation of the point at position $x_{i}$ of link $i$ for time $t$, where $x_{i} \in\left[0, L_{i}\right]$. Here, we use superscript "dot" to represent the derivative with respect to time variable $t$ and superscript "prime" to denote the derivative with respect to spatial variable $x_{i}$, i.e., $\quad \dot{u}_{1}\left(x_{1}, t\right)=\partial u_{1} / \partial t \quad$ and $u_{1}^{\prime}\left(x_{1}, t\right)=\partial u_{1} / \partial x_{1}$. Besides, subscript " $E$ " expresses variable $x_{i}$ equal to $L_{i}$, while subscript " 0 " expresses variable $x_{i}$ equal to 0 , i.e., $u_{1 E}=u_{1}\left(L_{1}, t\right)$ and $u_{10}=u_{1}(0, t)$. $\theta_{m i}$ denotes the angular position of joint $i$; $\theta_{i}$ is the angular position of link $i$. Notice that $\theta_{1}=\theta_{m 1}$ and $\theta_{2}=\theta_{m 2}+u_{1}^{\prime}\left(L_{1}\right)$. Since $u_{1}^{\prime}\left(L_{1}\right)$ is relatively small, this term can be ignored in the following derivations, leading to $\theta_{2}=\theta_{m 2}$ [22]. 


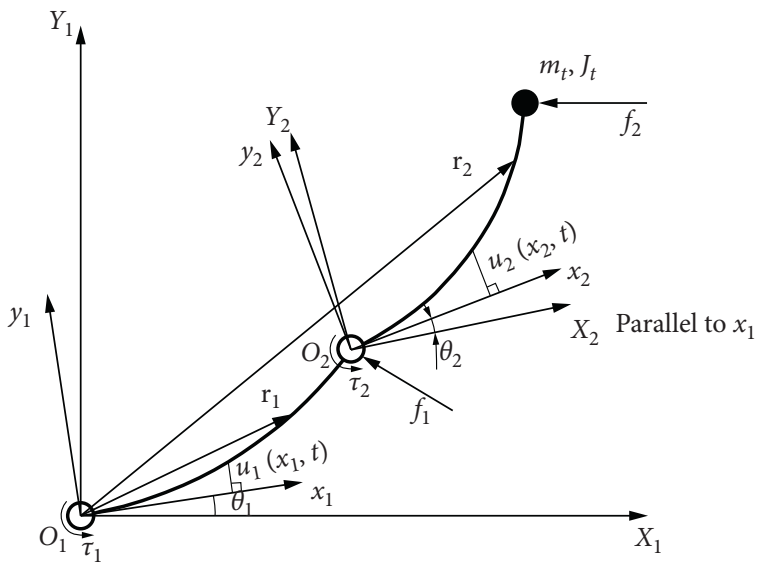

Figure 1: Sketch of the two-link flexible manipulator system.

To establish the dynamic model of the whole system, we apply Hamilton's principle as

$$
\int_{0}^{t} \delta(T-U+Q) \mathrm{d} t=0
$$

where $T$ is the kinetic energy of the system, $U$ is the totally potential energy, and $Q$ is the work by the nonconservative forces, i.e., the input torques and the actuator forces. The expressions of these three terms are given by equations (2)-(4):

$$
\begin{aligned}
T= & \frac{1}{2} \sum_{i=1}^{2}\left(\rho_{i} \int_{0}^{L_{i}} \dot{r}_{i}^{T}\left(x_{i}\right) \dot{r}_{i}\left(x_{i}\right) \mathrm{d} x_{i}\right) \\
& +\frac{1}{2} \sum_{i=1}^{2}\left(J_{m i} \dot{\theta}_{m i}^{2}+m_{i} \dot{p}_{i-1}^{T} \dot{p}_{i-1}\right) \\
& +\frac{1}{2}\left(m_{t} \dot{p}_{2}^{T} \dot{p}_{2}+\sum_{i=1}^{2}\left[J_{t i} \sum_{j=1}^{i}\left(\dot{\theta}_{j}+\dot{u}_{j E}^{\prime}\right)\right]^{2}\right), \\
U= & \sum_{i=1}^{2} \frac{1}{2} \int_{0}^{L_{i}} E I_{i}\left(u_{i}^{\prime \prime}\right)^{2} \mathrm{~d} x_{i}, \\
Q= & \sum_{i=1}^{2}\left(\tau_{i} \theta_{m i}+f_{i} z_{i E}\right),
\end{aligned}
$$

where $\mathbf{r}_{i}\left(x_{i}\right)=\left[\begin{array}{ll}x_{i} & u_{i}\left(x_{i}, t\right)\end{array}\right]^{T}$ and $\mathbf{p}_{i-1}=\mathbf{r}_{i}(0)$ represents the position vector of the $i$ th hub, while $\mathbf{p}_{2}=\mathbf{r}_{2}\left(L_{2}\right)$ and $u_{i E}^{\prime}=\partial u_{i} /\left.\partial x_{i}\right|_{x_{i}=L_{i}} \cdot z_{i}\left(x_{i}, t\right)$ is the arc length of flexible link $i$, which can be written as

$$
\begin{aligned}
& z_{1}\left(x_{1}\right)=x_{1} \theta_{1}+u_{1}\left(x_{1}\right) \\
& z_{2}\left(x_{2}\right)=x_{2}\left(\theta_{1}+\theta_{2}\right)+u_{2}\left(x_{2}\right)+\int_{.0}^{t}\left[\dot{z}_{1}\left(L_{1}, \xi\right) \cos \theta_{2}\right] d \xi
\end{aligned}
$$

where $x_{2}\left(\theta_{1}+\theta_{2}\right)$ means that the displacement in position $x_{2}$ induced by joint rotation of both link 1 and link $2, u_{2}\left(x_{2}\right)$ represents the transverse displacement of the flexible link, and $\int_{0}^{t}\left[\dot{z}_{1}\left(L_{1}, \xi\right) \cos \theta_{2}\right] d \xi$ denotes the displacement at $x_{2}$ induced by the motion of the end of link 1 . The integral term of equation (6) means that the displacement generated by the velocity of link 1 from time 0 to $t$. All vectors proposed above are in the global coordinate system.

Substituting equations (2)-(4) into equation (1) and applying integration by parts, we can obtain the dynamic model as well as boundary conditions of the flexible manipulator. To ensure that the boundary conditions only contain static boundary states, we further calculate the integrations in the above model with respect to spatial variable $x_{i}$ and neglect the higher-order terms such as $\sin \theta_{2} \dot{\theta}_{2}$ that are relatively small. After some necessary derivations, we can derive the dynamic model of the flexible manipulator as follows.

Equations of the flexible motion:

$$
\begin{gathered}
\rho_{1} \ddot{z}_{1}\left(x_{1}, t\right)+E I_{1} u_{1}^{(4)}\left(x_{1}, t\right)=0, \\
\rho_{2} \ddot{z}_{2}\left(x_{2}, t\right)+E I_{2} u_{2}^{(4)}\left(x_{2}, t\right)=0, \\
J_{m 1} \ddot{\theta}_{1}-E I_{1} u_{10}-\tau_{1}=0, \\
J_{t 1} J_{m 2} \ddot{\theta}_{2}-J_{m 2} E I_{1} u_{1 E}-J_{t 1} E I_{2} u_{2 E} \\
+J_{t 1} L_{1} f_{1}-\left(J_{t 1}+J_{m 2}\right) \tau_{2}=0 .
\end{gathered}
$$

Boundary conditions:

$$
\begin{array}{r}
x_{1}=0: u_{10}=u_{10}^{\prime}=0, \\
x_{1}=L_{1}: a(t) \ddot{z}_{1}\left(L_{1}\right)+\dot{a}(t) \dot{z}_{1}\left(L_{1}\right)+E I_{2} \dot{b}_{1}(t)+\dot{b}_{2}(t)-E I_{1} u_{1 E}^{\prime \prime \prime}-f_{1}=0, \\
\rho_{2} \dot{z}_{1 E}^{2} \sin \theta_{2} \cos \theta_{2}+E I_{2} \dot{z}_{1 E} b_{3}(t) \sin \theta_{2}+E I_{2} L_{2} u_{2 E}^{\prime \prime \prime}+E I_{2} u_{20}^{\prime \prime}-J_{t 1}\left(\ddot{\theta}_{1}+\ddot{u}_{1 E}^{\prime}\right) \\
-J_{m 2}\left(\ddot{\theta}_{1}+\ddot{\theta}_{2}+\ddot{u}_{1 E}^{\prime}\right)-m_{t} L_{2}\left(L_{2} \ddot{\theta}_{2}+\ddot{u}_{2 E}+\ddot{z}_{1 E} \cos \theta_{2}\right) \\
-m_{t} \dot{z}_{1 E} \sin \theta_{2}\left(L_{2} \dot{\theta}_{2}+\dot{u}_{2 E}\right)-E I_{1} u_{1 E}^{\prime \prime}=0,
\end{array}
$$




$$
\begin{gathered}
x_{2}=0: u_{20}=u_{20}^{\prime}=0, \\
x_{2}=L_{2}: m_{t} \ddot{z}_{2}\left(L_{2}\right)-E I_{2} u_{2 E}^{\prime \prime \prime}-f_{2}=0, \\
J_{t 2}\left(\ddot{\theta}_{1}+\ddot{\theta}_{2}+\ddot{u}_{1 E}^{\prime}+\ddot{u}_{2 E}^{\prime}\right)+E I_{2} u_{2 E}^{\prime \prime}=0,
\end{gathered}
$$

where the auxiliary functions above are defined as

$$
\begin{aligned}
& a(t)=\rho_{2} L_{2} \sin ^{2} \theta_{2}+m_{2}+m_{t} \sin ^{2} \theta_{2}, \\
& b_{1}(t)=\cos \theta_{2} \int_{0}^{t} u_{2}^{\prime \prime \prime}(0, \xi) d \xi, \\
& b_{2}(t)=\cos \theta_{2} \int_{0}^{t} f_{2}(\xi) d \xi, \\
& b_{3}(t)=E I_{2} \int_{0}^{t}\left[u_{2}^{\prime \prime \prime}\left(L_{2}, \xi\right)-u_{2}^{\prime \prime \prime}(0, \xi)\right] d \xi .
\end{aligned}
$$

From the above derivations, we can see that two PDEs (7) and (8) represent the vibration equations of the two flexible links, while ODEs (11)-(16) represent the system boundary conditions. Equations (9) and (10) represent the relation between the joint angle and its corresponding input torque of link 1 and link 2, respectively. Then, we design a boundary controller updated via the RBF NN algorithm in the followings. The input torques $\tau_{i}$ of the controller are used to track the angular positions, while the input forces $f_{i}$ are proposed to suppress the vibration of flexible manipulators simultaneously. Thus, the counteractive effect caused by only torque input control can be avoided well.

Remark 1. To make the subsequent control law derivation and stability analysis more convenient, we further facilitate the boundary conditions of two PDEs (7) and (8) by differentiating $z_{i}\left(x_{i}\right)$ with respect to $x_{i}$ :

$$
\begin{aligned}
& \frac{\partial z_{1}\left(x_{2}, t\right)}{\partial x_{1}}=\theta_{1}+\frac{\partial u_{1}\left(x_{1}, t\right)}{\partial x_{1}}, \\
& \frac{\partial z_{2}\left(x_{2}, t\right)}{\partial x_{2}}=\theta_{1}+\theta_{2}+\frac{\partial u_{2}\left(x_{2}, t\right)}{\partial x_{2}},
\end{aligned}
$$

when $t=0$, leading to

$$
\begin{aligned}
& z_{1}^{\prime}(0, t)=\theta_{1}, \\
& z_{2}^{\prime}(0, t)=\theta_{1}+\theta_{2},
\end{aligned}
$$

so that we can obtain that

$\frac{\partial^{n} z_{i}\left(x_{i}, t\right)}{\partial x_{i}^{n}}=\frac{\partial^{n} u_{i}\left(x_{i}, t\right)}{\partial x_{i}^{n}}$, for any $n \in Z \backslash\{1\}$, and $\forall x_{i} \in\left[0, L_{i}\right]$.

By means of deriving on both sides of equations (5) and (6) with respect to time, it is straightforward to derive the following initial conditions (25) and (26):

$$
\begin{aligned}
& \dot{z}_{1}(0, t)=0, \\
& \dot{z}_{2}(0, t)=\dot{z}_{1}\left(L_{1}, t\right) \cos \theta_{2} .
\end{aligned}
$$

Assumption 1. Given that the flexural displacements are sufficiently small, the transverse displacement $u_{i}\left(x_{i}\right)$ could be considered as a linear function of the spatial variable $x_{i}$, i.e.,

$$
u_{i}\left(x_{i}\right)=x_{i} u_{i}^{\prime}\left(x_{i}\right), \quad \forall x_{i} \in\left[0, L_{i}\right], i=1,2 .
$$

Property 1. Substituting Assumption 1 into the expressions of $z_{1}\left(L_{1}\right)$ and $z_{2}\left(L_{2}\right)$, respectively, then substituting equation (24) into the results, we can obtain the additional property as follows:

$$
\begin{aligned}
& \dot{z}_{1}^{\prime}\left(L_{1}\right)=\frac{\dot{z}_{1}\left(L_{1}\right)}{L_{1}}, \\
& \dot{z}_{2}^{\prime}\left(L_{2}\right)=\frac{\dot{z}_{2}\left(L_{2}\right)-\dot{z}_{1}\left(L_{1}\right) \cos \theta_{2}}{L_{2}} .
\end{aligned}
$$

\section{Adaptive Boundary Control Strategy}

3.1. Boundary Controller. In the proposed dynamic model above, the 2nd joint mass $m_{2}$ contains the total mass of the force actuator and the sensor which both install at the end of joint 1. Thus, $m_{2}$ is seen as uncertain in our study, too. Considering the parametric uncertainties above, i.e., the payload mass $m_{t}$ and the 2 nd joint mass $m_{2}$ whose values have much influence on the motion of flexible links, and we first propose a boundary control algorithm to track the angular positions and suppress the vibration. Then, the RBF NN is utilized in the design of the adaptive controller, so as to compensate for the multi-parameter uncertainty. We assume that the following parameters could be measured in practice or calculated by the backward difference approach: $\theta_{i}, \theta_{i}, \dot{u}_{i E}, u_{i 0}^{\prime \prime}$, $u_{i E}^{\prime \prime}, u^{\prime \prime \prime}{ }_{i 0}$, and $u^{\prime \prime \prime}{ }_{i E}$, for any $i=1,2$ (i.e., the above measurements are only changed with time $t)$. Note that $E I_{i} u^{\prime \prime \prime}{ }_{i E}$ represents the transverse internal force at the tip of link $i$.

Defining the position error of the joint angle as $e_{i}=\theta_{i}-\theta_{i d}$, we use the position error in the boundary control law to track the joint angles, where the subscript " $d$ " denotes the desired values. Thus, the issue is to design such a controller that the angular positions and elastic deformations could ensure that

$$
\theta_{i} \longrightarrow \theta_{i d}, u_{i}\left(x_{i}\right) \longrightarrow 0, \dot{u}_{i}\left(x_{i}\right) \longrightarrow 0 \text {, when, } t \longrightarrow \infty \text {. }
$$


Based on the form of boundary conditions (16), (17), (19), and (23) and the subsequent stability analysis shown in Section 4, we give the boundary control law as follows:

$$
\begin{aligned}
\tau_{1}= & -k_{1} e_{1}-k_{2} \dot{e}_{1}+E I_{2} u_{20}^{\prime \prime} \\
\tau_{2}= & -k_{3} e_{2}-k_{4} \dot{e}_{2}-\frac{J_{m 2} E I_{1}}{J_{t 1}+J_{m 2}} u_{1 E}^{\prime \prime} \\
& -\frac{J_{t 1} E I_{2}}{J_{t 1}+J_{m 2}} u_{2 E}^{\prime \prime}+\frac{J_{t 1} L_{1}}{J_{t 1}+J_{m 2}} f_{1}+E I_{2} u_{20}^{\prime \prime} \\
f_{1}= & -k_{5} \widehat{a}(t) \dot{z}_{1}\left(L_{1}\right)+E I_{2} \dot{b}_{1}(t)+\dot{b}_{2}(t) \\
& -\frac{E I_{1}}{L_{1}} u_{1 E}^{\prime \prime}+\frac{E I_{2}}{L_{2}} u_{2 E}^{\prime \prime} \cos \theta_{2}-E I_{2} u_{20}^{\prime \prime \prime} \cos \theta_{2} \\
f_{2}= & -k_{6} \dot{z}_{2}\left(L_{2}\right)-\frac{E I_{2}}{L_{2}} u_{2 E}^{\prime \prime}
\end{aligned}
$$

where $k_{1}, k_{2}, \ldots, k_{6}$ represent the control gains, which are all strictly positive constants. In equation (33), $\hat{a}(t)$ is the estimate value of the auxiliary function $a(t)$, which has the form of $\hat{a}(t)=\rho_{2} L_{2} \sin ^{2} \theta_{2}+\widehat{m}_{2}+\widehat{m}_{t} \sin ^{2} \theta_{2} . \widehat{m}_{2}$ and $\widehat{m}_{t}$ are the estimate values of $m_{2}$ and $m_{t}$, respectively. We assume that the uncertain parameters $\widehat{m}_{2}$ and $\widehat{m}_{t}$ are both bounded, satisfying $\widehat{m}_{2} \in\left[\underline{m}_{2}, \bar{m}_{2}\right]$ and $\widehat{m}_{t} \in\left[\underline{m}_{t}, \bar{m}_{t}\right]$. Therefore, $\widehat{a}(t)$ is also bounded. According to the boundary control law (31)-(34), we can see that the parametric uncertainties have important influence on the auxiliary function, which further influences the accuracy of the input force $f_{1}$ directly. Thus, considering the good nonlinear approximation ability of the RBF NN, we further utilize it to design an adaptive algorithm to approach the uncertain function $\widehat{a}(t)$.

3.2. RBF NN Adaptation Algorithm. Since the RBF NN has good ability to approximate nonlinear functions, it is utilized to compensate for the parametric uncertainty in this Section. In order to update the value of the estimate function $\widehat{a}(t)$ adaptively during the control strategy, here we use the real value of $a(t)$ as our objective function while the estimate value of $\widehat{a}(t)$ can be the output function of the RBF network. $\mathrm{RBF} N \mathrm{~N}$ is a forward network, using RBF as the basis of hidden neurons to form the hidden layer space. The structure of the proposed RBF NN is shown in Figure 2. It consists of three components, i.e., input layer, hidden layer, and output layer, respectively. The Gaussian function is chosen as the basis function in the RBF NN.

In Figure 2, we can see that the input activation is defined as $\mathbf{p}=\left[\begin{array}{llll}p_{1} & p_{2} & \cdots & p_{d}\end{array}\right]^{T}$, and the Gaussian function is defined as $\varphi_{j}\left(\left\|\mathbf{p}-\mathbf{c}_{\mathbf{j}}\right\|\right)=\exp \left(-\left\|\mathbf{p}-\mathbf{c}_{\mathbf{j}}\right\|^{2} / 2 \sigma^{2}\right)$. $\mathbf{c}_{\mathbf{j}}$ is the basis center vector, $\mathbf{c}_{\mathbf{j}}=\left[\begin{array}{lllll}c_{1} & c_{2} & \cdots & c_{d}\end{array}\right] ; \sigma$ represents the basis width of the $j$ th Gaussian function, $\sigma=\left[\begin{array}{llll}\sigma_{1} & \sigma_{2} & \cdots & \sigma_{d}\end{array}\right]^{T}$. Both $\mathbf{c}_{\mathbf{j}}$ and $\sigma$ have the same dimensions as the input p. $w_{j}$ represents the $j$ th element of weight vector $W$ so that

$$
W=\left[\begin{array}{llll}
w_{1} & w_{2} & \cdots & w_{d}
\end{array}\right]^{T} .
$$

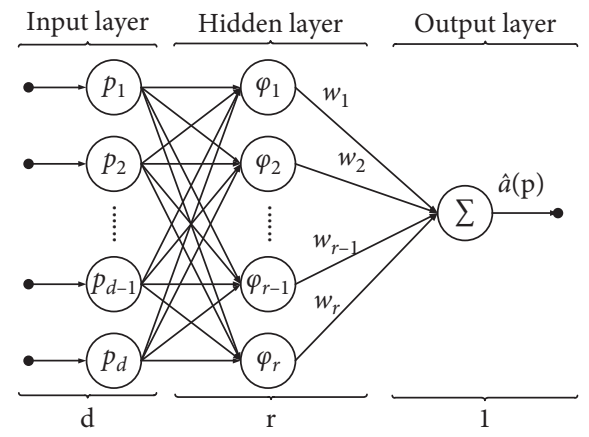

Figure 2: The structure of the designed RBF NN.

Define that the optimal weight matrix as

$$
W^{*}=\arg \min _{W \in \Omega}[\sup |a(\mathbf{p})-\hat{a}(\mathbf{p})|],
$$

where $\Omega$ is the set of $W$. Thus, the auxiliary function can be rewritten as $a=W^{* T} \Phi+\varepsilon$, where $\varepsilon$ is the network approximation error, $\|\varepsilon\| \leq \varepsilon_{N} \cdot \varepsilon_{N}$ is the given network approximation accuracy, satisfying $\varepsilon_{N}>0$. Therefore, the output of the network can be defined as the estimate value $\hat{a}$, which has the following expression:

$$
\widehat{a}(\mathbf{p})=\widehat{W}^{T} \boldsymbol{\Phi},
$$

where $\Phi=\left[\begin{array}{llll}\varphi_{1} & \varphi_{2} & \ldots & \varphi_{r}\end{array}\right]^{T}$ and $\widehat{W}$ is the estimate value of the weight vector $W$. Then, the estimate error of uncertain function is

$$
\begin{aligned}
& \tilde{a}(\mathbf{p})=a-\widehat{a}(\mathbf{p})=\left(W^{*}-\widehat{W}\right)^{T} \Phi+\varepsilon=\widetilde{W}^{T} \boldsymbol{\Phi}+\varepsilon, \\
& \dot{\tilde{a}}(\mathbf{p})=a-\widehat{a}(\mathbf{p})=-\dot{\hat{W}}^{T} \boldsymbol{\Phi} .
\end{aligned}
$$

The training process of the RBF NN is divided into three steps. (1) Determine the initial input and output vectors of the network. (2) Determine the basis centers and widths of the Gaussian function. (3) Determine the weight matrix $W$ between the hidden layer and the output layer. Since the end of each link has the largest amplitude of vibration, the performance index minimized by the $\mathrm{RBF} \mathrm{NN}$ is given as follows:

$$
\begin{aligned}
R(t) & =\frac{1}{2} \mathbf{e}^{T} \mathbf{e} \\
\mathbf{e} & =\left[e_{1}, e_{2}, \dot{e}_{1}, \dot{e}_{2}, u_{1 E}, u_{2 E}\right]^{T} .
\end{aligned}
$$

Here, we use the control error e as the input vector so that we have $d=6$. The weight matrix of the RBF NN is updated with the following adaptation law, which is given as

$$
\dot{\widehat{W}}=\left(\alpha \widetilde{a}+\beta k_{5} \dot{z}_{1 E}^{2}\right) \Phi
$$

where $\beta$ is the learning rate of the network and $0<\beta<1$. Here, we introduce the parameter $\alpha$ to offset the approximation error of the network and $\alpha>0$. Substituting the RBF $\mathrm{NN}$ adaptive law (41) into the boundary control law 
(31)-(34), we can derive the adaptation closed-loop control system as well as Theorem 1, which is shown in Section 4. It means that no offline training or learning process is needed for the designed RBF NN and the approximation could be completed during the operation of the flexible manipulator. To make the consequent stable analysis more clear, here we introduce Lemma 1 [24] as follows.

Lemma 1. Suppose there are two matrices $\mathbf{X}_{1}$ and $\mathbf{X}_{2}$, satisfying $\mathbf{X}_{1} \in \mathbb{R}^{n \times 1}$ and $\mathbf{X}_{2} \in \mathbb{R}^{n \times 1}$; then, we have

$$
\mathbf{X}_{1}^{T} \mathbf{X}_{2} \leq \frac{1}{2}\left\|\mathbf{X}_{1}\right\|^{2}+\frac{1}{2}\left\|\mathbf{X}_{2}\right\|^{2}
$$

\section{Stability Analysis}

Theorem 1. The combined adaptive control law (31)-(34) with the adaptation law (41) can ensure that the asymptotic stability and uniform convergence of the closed-loop system, i.e.,

$$
\theta_{i} \longrightarrow \theta_{i d}, u_{i}\left(x_{i}\right) \longrightarrow 0, \dot{u}_{i}\left(x_{i}\right) \longrightarrow 0, \text { when } t \longrightarrow \infty \text {, for any } x_{i} \in\left[0, L_{i}\right], \quad i=1,2
$$

Proof. To prove the asymptotic stability of the adaptive boundary controller, we use the Lyapunov direct approach. Combined with LaSalle's invariance principle, here the Lyapunov direct approach is extended to infinite-dimensional space [25]. Different from Ref. [6], the tip mass $m_{2}$ of link 1 is also uncertain in this paper. Hence, the combined uncertain function is utilized to describe the co-uncertainty of the two parameters. We need to prove that the proposed $\mathrm{RBF} N \mathrm{~N}$ adaptive law can ensure that the uncertain function will converge to its optimal value, i.e., the estimate error can converge to zero as time $t \longrightarrow \infty$. Substituting the control law (31)-(34) into equations (9), (10), (12), and (15), we can derive the closed-loop dynamics of the system:

$$
\begin{array}{r}
\ddot{\theta}_{1}=\frac{1}{J_{m 1}}\left(E I_{1} u_{10}^{\prime \prime}+E I_{2} u_{20}^{\prime \prime}-k_{1} e_{1}-k_{2} \dot{e}_{1}\right), \\
\ddot{\theta}_{2}=\frac{J_{t 1}+J_{m 2}}{J_{m 2} J_{t 1}}\left(E I_{2} u_{20}^{\prime \prime}-k_{3} e_{2}-k_{4} \dot{e}_{2}\right), \\
a(t) \ddot{z}_{1}\left(L_{1}\right)+\dot{a}(t) \dot{z}_{1}\left(L_{1}\right)=r(t), \\
\ddot{z}_{2}\left(L_{2}\right)=\frac{1}{m_{t}}\left[-k_{6} \dot{z}_{2}\left(L_{2}\right)-\frac{E I_{2}}{L_{2}} u_{2 E}^{\prime \prime}+E I_{2} u_{2 E}^{\prime \prime}\right],
\end{array}
$$

$$
\begin{aligned}
r(t)= & E I_{1} u_{1 E}^{\prime \prime \prime}-k_{5} a(t) \dot{z}_{1}\left(L_{1}\right)-\frac{E I_{1}}{L_{1}} u_{1 E}^{\prime \prime}+\frac{E I_{2}}{L_{2}} u_{2 E}^{\prime \prime} \cos \theta_{2} \\
& -E I_{2} u_{20}^{\prime \prime \prime} \cos \theta_{2}+k_{5} \tilde{a}(t) \dot{z}_{1}\left(L_{1}\right) .
\end{aligned}
$$

Substituting the expressions of $z_{1}\left(x_{1}\right)$ and $z_{2}\left(x_{2}\right)$ into equations (7) and (8), respectively, then putting (44) and (45) into the results, we can obtain

$$
\ddot{u}_{1}\left(x_{1}\right)=-\frac{E I_{1}}{\rho_{1}} u_{1}^{(4)}-\frac{x_{1}}{J_{m 1}}\left(E I_{1} u_{10}^{\prime \prime}+E I_{2} u_{20}^{\prime \prime}-k_{1} e_{1}-k_{2} \dot{e}_{1}\right) \text {, }
$$

$$
\begin{aligned}
\ddot{u}_{2}\left(x_{2}\right)= & -\frac{E I_{2}}{\rho_{2}} u_{2}^{(4)}-\frac{x_{2}}{J_{m 1}}\left(E I_{1} u_{10}^{\prime \prime}+E I_{2} u_{20}^{\prime \prime}-k_{1} e_{1}-k_{2} \dot{e}_{1}\right) \\
& -\frac{J_{t 1}+J_{m 2}}{J_{t 1} J_{m 2}}\left(E I_{2} u_{20}^{\prime \prime}-k_{3} e_{2}-k_{4} \dot{e}_{2}\right) x_{2} \\
& -\frac{r(t)-\dot{a}(t) \dot{z}_{1}\left(L_{1}\right)}{a(t)} \cos \theta_{2}+\dot{z}_{1}\left(L_{1}\right) \sin \theta_{2} \dot{\theta}_{2}
\end{aligned}
$$

To transfer the closed-loop system into the state space, we define the following variables of state space as

where

$$
\begin{aligned}
& \mathbf{q}_{0}=\left[\begin{array}{llllllllll}
q_{1} & q_{2} & q_{3} & q_{4} & q_{5} & q_{6} & q_{7} & q_{8} & q_{9} & q_{10}
\end{array}\right]^{T},
\end{aligned}
$$

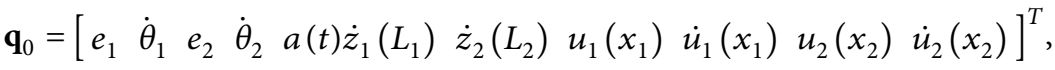

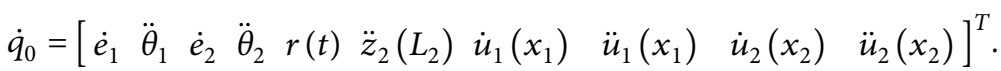

Introducing a state vector $\mathbf{q}=\left[\begin{array}{ll}\mathbf{q}_{0} & q_{11}\end{array}\right]^{T}$, we can rewrite equations (46)-(50) in the following compact form:

$$
\begin{array}{r}
\dot{q}=\mathscr{A} \mathbf{q}+f(\mathbf{q}), \\
\mathbf{q}(0) \in \mathscr{H},
\end{array}
$$

where vector $\mathbf{q}$ is defined in the Hilbert space $\mathscr{H}$, and $\mathscr{H}=\mathbb{R}^{6} \times H^{2} \times L^{2} \times H^{2} \times L^{2} \times \mathbb{R} . \mathscr{A}$ represents the infinite linear operator, i.e.,

$$
\mathscr{A}=\left[\begin{array}{cc}
\mathscr{A}_{0} & 0 \\
0 & \omega
\end{array}\right]
$$


where $\omega \in \mathbb{R}$ is a positive constant. $\mathscr{A}_{0}$ represents an infinitedimensional linear operator defined as

$$
\begin{aligned}
\mathscr{A}_{0} \mathbf{q}_{0} & =\left[\begin{array}{llllllllll}
q_{2} & \vartheta_{1} & q_{4} & \vartheta_{2} & \vartheta_{3} & \vartheta_{4} & q_{8} & \vartheta_{5} & q_{10} & \vartheta_{6}
\end{array}\right]^{T}, \quad \forall q_{0} \in D\left(\mathscr{A}_{0}\right), \\
f(\mathbf{q}) & =\left[\begin{array}{lllllllllll}
0 & 0 & 0 & 0 & h_{1} & 0 & 0 & 0 & 0 & h_{2} & h_{3}
\end{array}\right]^{T},
\end{aligned}
$$

where

$$
\begin{aligned}
h_{1}= & \frac{E I_{2}}{L_{2}} q_{9}^{\prime \prime}\left(L_{2}\right) \cos \theta_{2}+\frac{k_{5} q_{11} q_{5}}{a(t)}-E I_{2} q_{9}^{\prime \prime \prime}(0) \cos \theta_{2}, \\
h_{2}= & \frac{\cos \theta_{2}}{a(t)} s(t)+\frac{q_{5}}{a(t)} q_{4} \sin \theta_{2}, \\
h_{3}= & -\omega q_{11}-\left(\alpha q_{11}+\beta k_{5} \frac{q_{5}}{a(t)}\right) \boldsymbol{\Phi}^{\mathrm{T}} \boldsymbol{\Phi}, \\
s(t)= & \dot{a}(t) \frac{q_{5}}{a(t)}-E I_{1} q_{7}^{\prime \prime \prime}\left(L_{1}\right)+k_{5} q_{5}+\frac{E I_{1}}{L_{1}} q_{7}^{\prime \prime}\left(L_{1}\right) \\
& -\frac{E I_{2}}{L_{2}} q_{9}^{\prime \prime}\left(L_{2}\right) \cos \theta_{2}+E I_{2} q_{9}^{\prime \prime \prime}(0) \cos \theta_{2}-k_{5} q_{11} \frac{q_{5}}{a(t)} .
\end{aligned}
$$

Consider the boundary conditions in equations (11) and (14), the domain of the linear operator $\mathscr{A}_{0}$ and $\mathscr{A}$ are

$$
\begin{aligned}
D\left(\mathscr{A}_{0}\right)= & \left\{q \in \mathbb{R}^{6} \times H^{4} \times H^{2} \times H^{4}\right. \\
& \left.\times H^{2} \mid q_{7}(0)=0, q_{7}^{\prime}(0)=0, q_{9}(0)=0, q_{9}^{\prime}(0)=0\right\},
\end{aligned}
$$

$$
D(\mathscr{A})=D\left(\mathscr{A}_{0}\right) \times \mathbb{R},
$$

where

$$
\begin{aligned}
L^{2}(\Omega) & =\left\{\left.f\left|\int_{\Omega}\right| f(x)\right|^{2} d x<+\infty\right\}, \\
H^{k}(\Omega) & =\left\{f \mid f, f^{\prime}, f^{\prime \prime}, \cdots, f^{(k)} \in L^{2}(\Omega)\right\}, \Omega=\left[0, L_{i}\right] .
\end{aligned}
$$

Then, the Lyapunov function of the whole system is defined as

$$
V=V_{1}+V_{2}+V_{a},
$$

where

$$
\begin{aligned}
& V_{1}=\sum_{i=1}^{2} \frac{1}{2} \int_{0}^{L_{i}} \rho_{i} \dot{z}_{i}^{2}\left(x_{i}\right) d x_{i}+\sum_{i=1}^{2} \frac{1}{2} \int_{0}^{L_{i}} E I_{i}\left[z_{i}\left(x_{i}\right)\right]^{2} d x_{i} \\
& V_{2}=\frac{1}{2} J_{m 1} \dot{e}_{2}^{1}+\frac{J_{m 2} J_{t 1}}{2\left(J_{m 2}+J_{t 1}\right)} \dot{e}_{2}^{2}+\frac{1}{2} m_{t} \dot{z}_{2}^{2}\left(L_{2}\right)+\frac{1}{2} a(t) \dot{z}_{1}^{2}\left(L_{1}\right),
\end{aligned}
$$

$$
V_{a}=\frac{1}{2} k_{1} e_{1}^{2}+\frac{1}{2} k_{3} e_{2}^{2}+\frac{1}{2} \alpha \tilde{a}^{2}+\frac{1}{2 \beta} \widetilde{W}^{T} \widetilde{W} .
$$

Taking into account of the approximation error $\widetilde{a}$, we therefore design term $1 / 2 \alpha \widetilde{a}^{2}$ in the Lyapunov function. Note that $\widetilde{W}=W^{*}-\widehat{W}$ and $\dot{\bar{W}}=-\dot{W}$. According to Lemma 1 , we have

$$
\frac{1}{2 \beta} \widetilde{W}^{T} \widetilde{W} \leq \frac{1}{2 \beta^{2}}\|\widetilde{W}\|^{2} .
$$

We can see that $V$ is obviously positive definite. Furthermore, we need to guarantee the stability of the Lyapunov function. Differentiating $V$ with respect to time and then substituting equations (41)-(46) and boundary conditions (22)-(29) into the result, we can obtain the derivative of the Lyapunov function:

$$
\begin{aligned}
\dot{V}= & -k_{2} \dot{e}_{1}^{2}-k_{4} \dot{e}_{2}^{2}-\left[k_{5} a(t)+\frac{1}{2} \dot{a}(t)\right] \dot{z}_{1 E}^{2}-k_{6} \dot{z}_{2 E}^{2} \\
& +k_{5} \tilde{a} \dot{z}_{1 E}^{2}+\alpha \tilde{a} \dot{\tilde{a}}-\frac{1}{\beta}\left(W^{*}-\widehat{W}\right)^{T} \dot{\hat{W}} .
\end{aligned}
$$

Since the network error $\varepsilon$ is bounded and the RBF control parameters satisfy $\alpha \gg \beta$, substituting (38) and (39) into equation (76), we obtain

$$
\begin{aligned}
\dot{V} \leq & -k_{2} \dot{e}_{1}^{2}-k_{4} \dot{e}_{2}^{2}-\left[k_{5} a(t)+\frac{1}{2} \dot{a}(t)\right] \dot{z}_{1 E}^{2}-k_{6} \dot{z}_{2 E}^{2} \\
& -\left(W^{*}-\widehat{W}\right)^{T}\left(\frac{1}{\beta} \dot{\hat{W}}-k_{5} \dot{z}_{1 E}^{2} \boldsymbol{\Phi}-\frac{\alpha}{\beta} \widetilde{a} \mathbf{\Phi}\right) .
\end{aligned}
$$

Substituting the RBF NN adaptive law (41) into equations (71) and properly choosing $k_{5}$ satisfies

$$
k_{5}>\frac{\rho_{2} L_{2}+\bar{m}_{t}}{2 \underline{m}_{2}},
$$

where $\bar{m}_{t}$ is the maximum value of the estimate mass of the payload and $\underline{m}_{2}$ is the minimum mass of the joint $\mathrm{O}_{2}$. Then, we can guarantee that

$$
V>0 \text { and } \dot{V} \leq 0 .
$$

Equation (73) shows that $V$ is a bounded function and the energy of the whole system is dissipated. Based on LumerPhilips theorem, $\dot{V} \leq 0$ represents that the operator $\mathscr{A}$ is dissipative. Then, we prove that there exists $\lambda>0$ satisfying range $(\lambda I-\mathscr{A}) \in \mathscr{H}$.

Define $\mathbf{y}=\left[\begin{array}{lllllllllll}y_{1} & y_{2} & y_{3} & y_{4} & y_{5} & y_{6} & y_{7} & y_{8} & y_{9} & y_{10} & y_{11}\end{array}\right]^{T}$, set

$$
\mathscr{A} \mathbf{q}=\mathbf{y},
$$

where 


$$
\begin{aligned}
y_{1}= & q_{2}, \\
y_{2}= & \frac{1}{J_{m 1}}\left[E I_{1} q_{7}(0)+E I_{2} q_{9}(0)-k_{1} q_{1}-k_{2} q_{2}\right], \\
y_{3}= & q_{4}, \\
y_{4}= & \frac{J_{t 1}+J_{m 2}}{J_{m 2} J_{t 1}}\left[E I_{2} q_{9}(0)-k_{3} q_{3}-k_{4} q_{4}\right], \\
y_{5}= & E I_{1} q^{\prime \prime \prime}{ }_{7}\left(L_{1}\right)-k_{5} q_{5}-\frac{E I_{1}}{L_{1}} q_{7}\left(L_{1}\right), \\
y_{6}= & \frac{1}{m_{t}}\left[-k_{6} q_{6}-\frac{E I_{2}}{L_{2}} q_{9}^{\prime \prime}\left(L_{2}\right)+E I_{2} q_{9}^{\prime \prime}\left(L_{2}\right)\right], \\
y_{7}= & q_{8}, \\
y_{8}= & -\frac{E I_{1}}{\rho_{1}} q_{7}^{(4)}\left(x_{1}\right)-\frac{x_{1}}{J_{m 1}}\left[E I_{1} q_{7}(0)+E I_{2} q_{9}(0)-k_{1} q_{1}-k_{2} q_{2}\right], \\
y_{9}= & q_{10}, \\
y_{10}= & -\frac{E I_{2}}{\rho_{2}} q_{9}^{(4)}\left(x_{1}\right)-\frac{x_{2}}{J_{m 1}}\left[E I_{1} q_{7}(0)+E I_{2} q_{9}(0)-k_{1} q_{1}-k_{2} q_{2}\right], \\
& -\frac{x_{2}\left(J_{m 2}+J_{t 1}\right)}{J_{m 2} J_{t 1}}\left[E I_{2}{ }^{\prime \prime} q_{9}(0)-k_{3} q_{3}-k_{4} q_{4}\right], \\
y_{11}= & \omega q_{11} .
\end{aligned}
$$

From the equations of state space above, we can solve the vector $\mathbf{q}$ by integration. Thus, the abstract equation $\mathscr{A} \mathbf{q}=\mathbf{y}$ has a unique solution $\mathbf{q} \in D(\mathscr{A})$, which means that there exists $\mathscr{A}^{-1}$ that maps $\mathscr{H}$ into $\mathbb{R}^{6} \times H^{4} \times H^{2} \times H^{4} \times H^{2} \times \mathbb{R}$. Since the mapping from $\mathbf{q} \in D(\mathscr{A})$ into $\mathbf{y} \in \mathscr{H}$ is compact, we can obtain that the operator $\mathscr{A}$ is also compact. Considering the equation,

$$
(\lambda I-\mathscr{A}) \mathbf{q}=\mathscr{A}\left(\lambda \mathscr{A}^{-1}-I\right) \mathbf{q}=\mathbf{y} .
$$

Based on contraction mapping principle, when $0<\lambda<\left\|\mathscr{A}^{-1}\right\|^{-1}$, equation (76) has a unique solution $\mathbf{q} \in D(\mathscr{A})$. That means operator $(\lambda I-\mathscr{A}) \in \mathscr{H}$ holds for any $\lambda>0$. According to Lumer-Phillips principle, operator $\mathscr{A}$ can generate a $C_{0^{-}}$-semigroup in $\mathscr{H}$. Furthermore, since $\mathscr{A}$ is a compact operator, the spectrum of $\mathscr{A}$ consists of isolated eigenvalues completely. For any $\lambda$ belongs to the resolvent set of $\mathscr{A}$, the operator $(\lambda I-\mathscr{A})^{-1}$ is compact. Thus, the solution trajectories of (54) are precompact in $\mathscr{H}$.

Now, we can conclude that the following three conditions hold: (1) the energy of system (54) is dissipative; (2) the operator $\mathscr{A}$ can generate a $C_{0}$-semigroup in Hilbert space $\mathscr{H}$; (3) the solution trajectories of the system are precompact in $\mathscr{H}$. According to LaSalle's invariance principle, the control system (54) is asymptotically stable if $\dot{V} \equiv 0$ could derive $\mathbf{q}=0$.
Let $\dot{V} \equiv 0$; we have $\dot{e}=\dot{z}_{1 E} \equiv 0$ and $\ddot{e}_{i}=\ddot{z}_{i E} \equiv 0$, i.e., $\dot{\theta}_{i}=0, \ddot{\theta}_{i}=0$, and $\ddot{u}_{i E}=0$. Substituting the results into the flexible motion equation, we can obtain

$$
\begin{gathered}
\rho_{i} \ddot{u}_{i}\left(L_{i}, t\right)+E I_{i} u_{i}^{(4)}\left(L_{i}, t\right)=0, \\
u_{i}^{(4)}\left(x_{i}, t\right)=-\frac{\rho_{i}}{E I_{i}} \ddot{u}_{i}\left(x_{i}, t\right) .
\end{gathered}
$$

From (77), we can derive that $u_{i}^{(4)}\left(L_{i}, t\right)=0$. Based on the variable separation method, let the solution be $u_{i}\left(x_{i}, t\right)=U_{i}\left(x_{i}\right) \cdot T(t)$; then, the transversal deformations of the two flexible links can be written as

$$
\frac{U_{i}^{(4)}\left(x_{i}\right)}{U\left(x_{i}\right)}=-\frac{\rho_{i}}{E I_{i}} \frac{\ddot{T}(t)}{T(t)}=\mu,
$$

i.e., $U_{i}^{(4)}\left(x_{i}\right)-\mu \ddot{X}=0$. Substituting the boundary conditions (11), (15), and (17), we can get the solution of equation (78) that $U_{i}\left(x_{i}\right)=0$, which means $\mathbf{q}=0$. Thus, the system performance can be asymptotically stabilized with properly choosing the control parameters, leading to the tracking error $e_{i} \longrightarrow 0, \quad u_{i}\left(x_{i}\right) \longrightarrow 0$, and $\quad \dot{u}_{i}\left(x_{i}\right) \longrightarrow 0$ as $t \longrightarrow \infty$. 


\section{Numerical Simulation}

In the simulation, the performance of the dynamic system under the proposed adaptive boundary control law is analyzed. We use the finite difference approach in this Section to solve the closed-loop control equations in form of PDEs. Spatial central difference and time forward difference are used in the simulation. The time step and space step are set as $\Delta t=5 \times 10^{-4} \mathrm{~s}$ and $\Delta x=6 \times 10^{-2} \mathrm{~m}$, respectively. The nominal parameters of two flexible links and the rigid payload are given as

$$
\begin{aligned}
L_{1} & =0.6 \mathrm{~m}, \\
L_{2} & =0.8 \mathrm{~m}, \\
E I_{1} & =3.5 \mathrm{Nm}^{2}, \\
E I_{2} & =4 \mathrm{Nm}^{2}, \\
\rho_{1} & =\rho_{2} \\
J_{m 1} & =0.2 \mathrm{Kg} \cdot \mathrm{m}^{2}, \\
J_{m 2} & =0.2 \mathrm{Kg} \cdot \mathrm{m}^{2}, \\
J_{t 1} & =0.6 \mathrm{Kg} \cdot \mathrm{m}^{2}, \\
J_{t 2} & =2 \mathrm{Kg} \cdot \mathrm{m}^{2}, \\
m_{1} & =0.55 \mathrm{Kg}, \\
m_{2} & =0.65 \mathrm{Kg}, \\
m_{t} & =2 \mathrm{Kg}, \\
\theta_{1 d} & =0.5 \mathrm{rad}, \\
\theta_{2 d} & =1 \mathrm{rad} .
\end{aligned}
$$

The initial values including the flexible manipulator state and the velocity of joint angles are all set to be zeros. Besides, the parameters used in the control algorithm are presented as $k_{1}=600, k_{2}=350, k_{3}=600, k_{4}=520, k_{5}=10$, and $k_{6}=8$. The parameters used in the RBF NN are $\alpha=7.3$ and $\beta=0.21$. So that the stable conditions derived in Section 5 can be satisfied, i.e.,

$$
\begin{gathered}
\alpha>>\beta, \\
k_{5}=10>\frac{\rho_{2} L_{2}+\bar{m}_{t}}{2 \underline{m}_{2}}=\frac{0.2 \times 0.8+2.5}{2 \times 0.58}=2.29 .
\end{gathered}
$$

We design 6 neurons in the hidden layer in the form of Gaussian function, where the initial values of the weight matrix, basis width vector, and center value matrix are shown as follows:

$$
\begin{aligned}
W & =\left[\begin{array}{llllll}
0.05 & 0.05 & 0.05 & 0.05 & 0.05 & 0.05
\end{array}\right]^{T}, \\
\boldsymbol{\sigma} & =10^{3} \times\left[\begin{array}{llllll}
2 & 2 & 2 & 2 & 2 & 2
\end{array}\right]^{T},
\end{aligned}
$$

$$
\mathbf{c}=\left[\begin{array}{llllll}
1 & 1 & 0 & 0 & 1 & 1 \\
2 & 1 & 0 & 0 & 1 & 2 \\
1 & 1 & 0 & 0 & 3 & 2 \\
3 & 1 & 1 & 2 & 0 & 1 \\
2 & 1 & 2 & 2 & 1 & 0 \\
1 & 1 & 0 & 0 & 2 & 1
\end{array}\right]^{T}
$$

The simulation results are demonstrated in Figures 3-8. Figure 3 shows the two actual joint angles $\theta_{1}$ and $\theta_{2}$; Figure 4 compares the estimate value of the RBF NN and the real value of the auxiliary function. Figure 5 represents the flexural displacement $u_{1}\left(x_{1}, t\right)$, while Figure 6 shows the flexural displacement $u_{2}\left(x_{2}, t\right)$; Figure 7 shows the two input torques applied at the joints; Figure 8 shows the two input forces applied at the end of link 1 and link 2.

From Figures 3, 5, and 6, we can see that the joint position errors of the two links converge to zero while the flexural displacements are also suppressed under the combined adaptive control law. Figures 5 and 6 show that the vibration during the motion of the links is small, while the peak values of our method are slightly larger. That means the vibration is suppressed well. The larger peak value in the process is generated by the energy concentration of the control system, leading to less energy in vibration. Figure 3 shows that the RBF NN adaptive algorithm can enable the uncertain function approach to its real value if the parameters are chosen properly. Besides, it takes about $1 \mathrm{~s}$ to track the uncertain function, realizing effective compensation for uncertain function. However, the approximation results of the RBF NN are sensitive to the initial values, which have an important influence on the compensation accuracy. The simulation results are in accordance with the theoretical analysis carried out in Section 4, which illustrates that the validity and effectiveness of the proposed adaptive combinational control law with RBF NN.

To further validate the effectiveness of our control method, we use another dynamic model derived in Ref. [6] and compare our work with the result of pure boundary control method. The relevant simulation parameters are listed in Tables 1 and 2.

The true value of the uncertain end mass $m_{t}$ is $2 \mathrm{Kg}$. Through Tables 1 and 2, we can see that $k_{5}$ in our control algorithm should satisfy $k_{5}>\rho_{2} L_{2}+\bar{m}_{t} / 2 \underline{m}_{2}$. So, we carefully adjust the control gains in our algorithm and choose $k_{5}=10$. By properly tuning the RBF NN to approach the uncertain function $\widehat{a}(t)$, we can have the following simulation results.

Compared with pure boundary control proposed in Ref. [6], we can see that the combined boundary control method with RBF NN converges faster, using less than $5 s$ to up to its real value. Figures 9(c) and 9(d) show the residual vibration of the two flexible links during the flexible motion is also effectively suppressed. As for the multiparameter uncertainty, our RBF NN coincides with the uncertain function from $3.2 \mathrm{~s}$ to $15 \mathrm{~s}$, while the pure boundary condition consumes about $6 \mathrm{~s}$. The comparison above means that our combinational control law has a faster learning ability under same accuracy. 

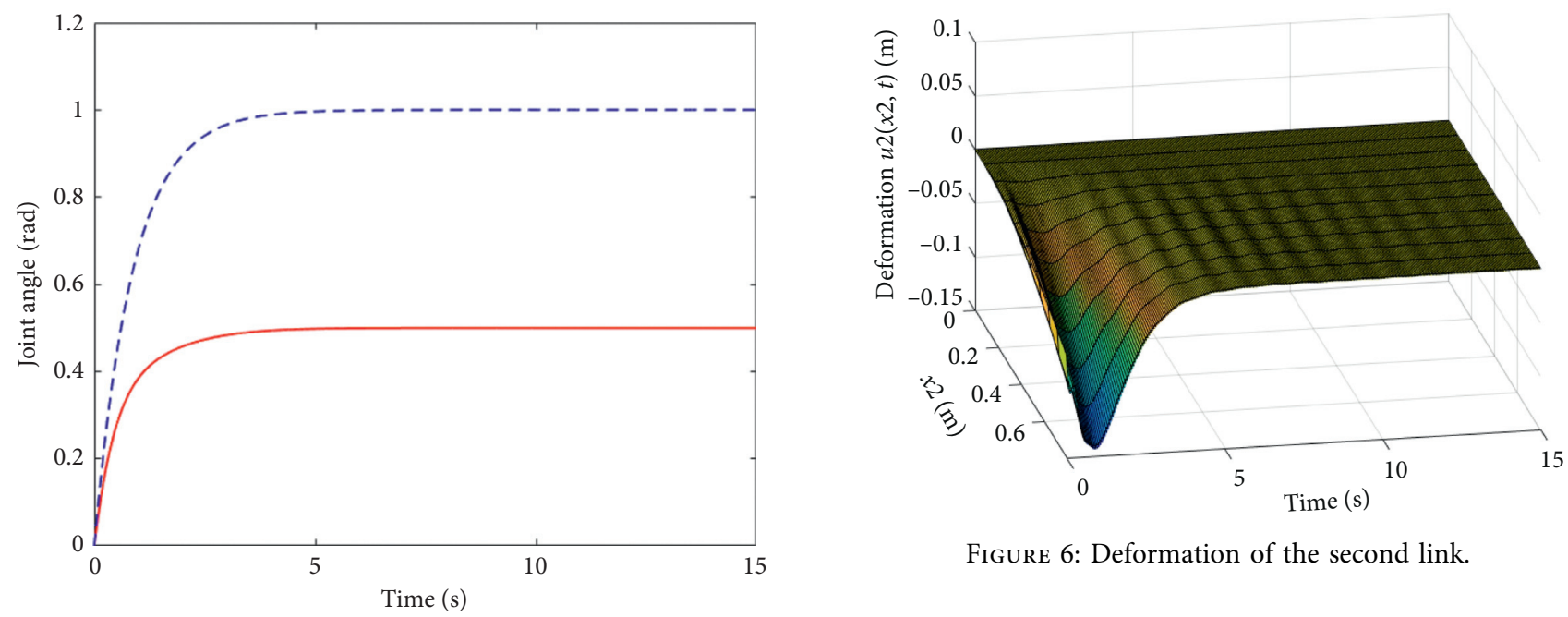

Figure 6: Deformation of the second link.

$-\theta 1$

Figure 3: Actual joint angles.
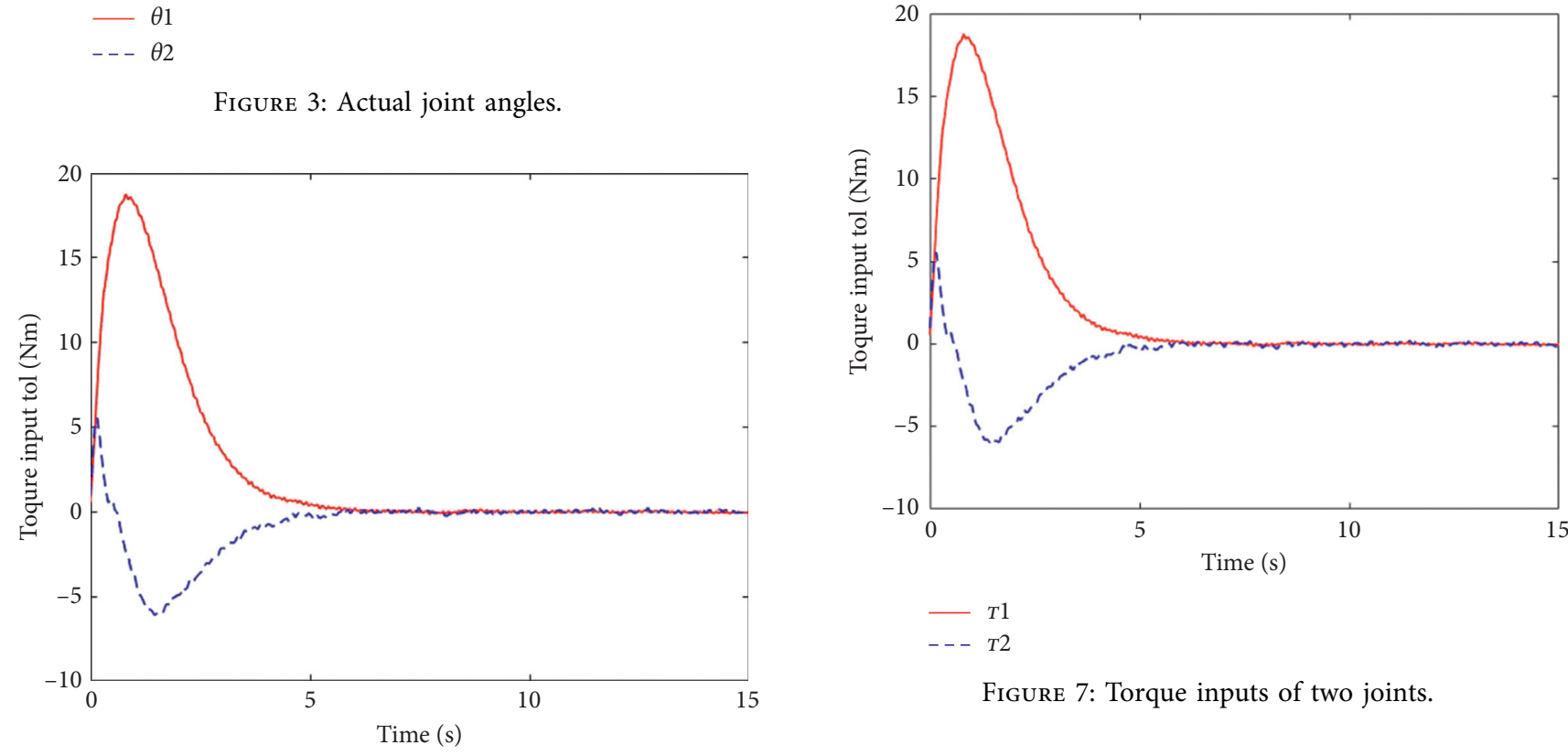

Figure 7: Torque inputs of two joints.

- $T 1$

$---T 2$

FIGURE 4: Uncertain function $a$ and its estimate $s$.

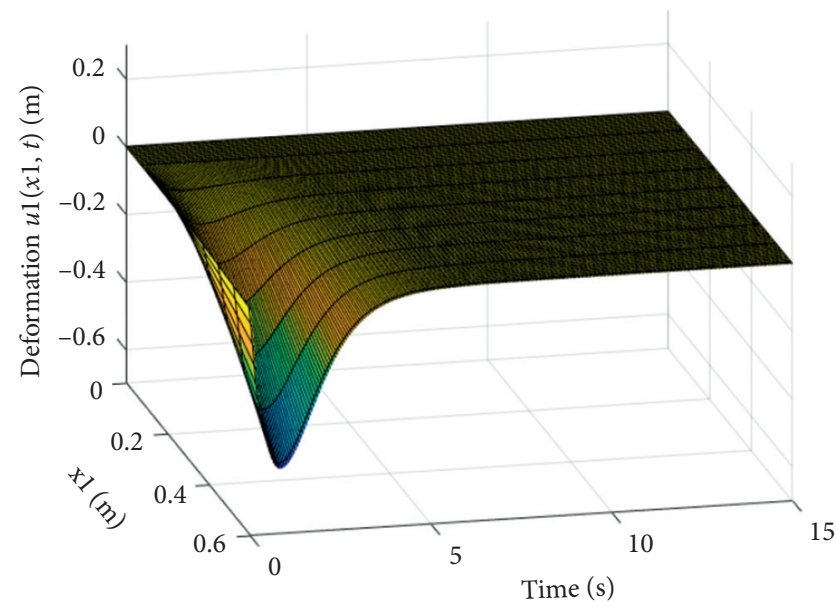

FIgURE 5: Deformation of the first link.

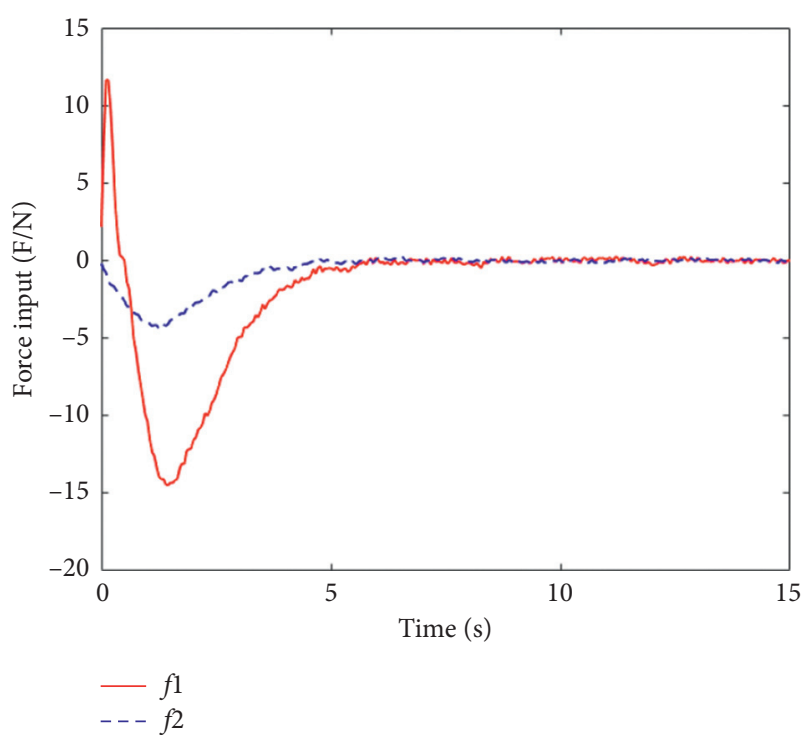

FIgURE 8: Force inputs of two joints. 
TABLE 1: The parameters used to compare with pure boundary control method. Parameters of link 1.

\begin{tabular}{lcccccc}
\hline$\rho_{1}(\mathrm{~kg} / \mathrm{m})$ & $L_{1}(\mathrm{~m})$ & $E I_{1}\left(\mathrm{Nm}^{2}\right)$ & $J_{m 1}\left(\mathrm{Kg} \cdot \mathrm{m}^{2}\right)$ & $J_{t 1}\left(\mathrm{Kg} \cdot \mathrm{m}^{2}\right)$ & $m_{1}(\mathrm{Kg})$ & $\theta_{1 d}(\mathrm{rad})$ \\
\hline 0.2 & 0.6 & 2 & 0.05 & 0.1 & 0.6 & 0.5 \\
\hline
\end{tabular}

TABLE 2: The parameters used to compare with the pure boundary control method. Parameters of link 2.

\begin{tabular}{lcccccc}
\hline$\rho_{2}(\mathrm{~kg} / \mathrm{m})$ & $L_{2}(\mathrm{~m})$ & $E I_{2}\left(\mathrm{Nm}^{2}\right)$ & $J_{m 2}\left(\mathrm{Kg} \cdot \mathrm{m}^{2}\right)$ & $J_{t 2}\left(\mathrm{Kg} \cdot \mathrm{m}^{2}\right)$ & $m_{2}(\mathrm{Kg})$ & $\theta_{2 d}(\mathrm{rad})$ \\
\hline 0.2 & 0.6 & 4 & 0.06 & 2 & 0.7 & 1 \\
\hline
\end{tabular}

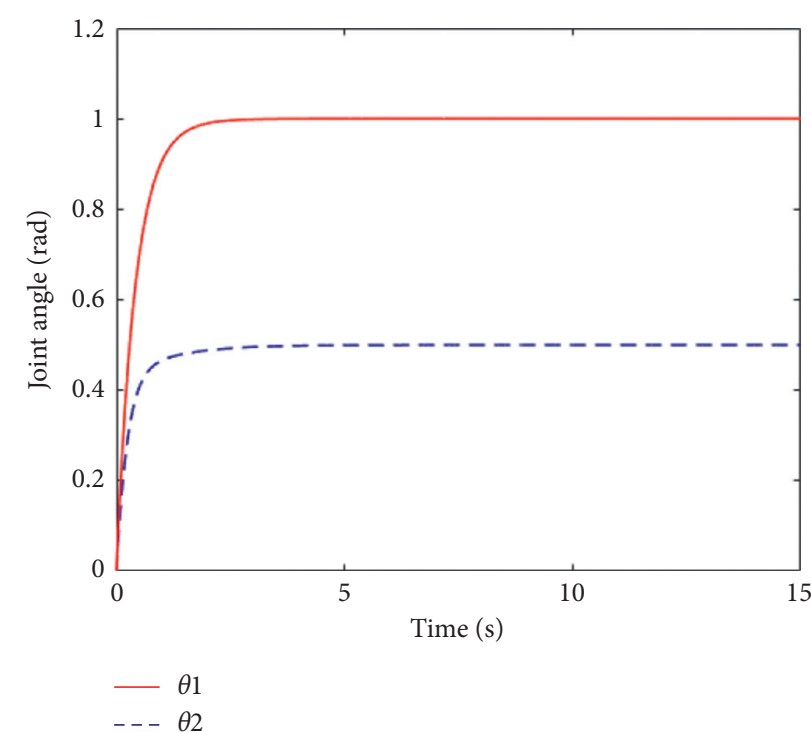

(a)

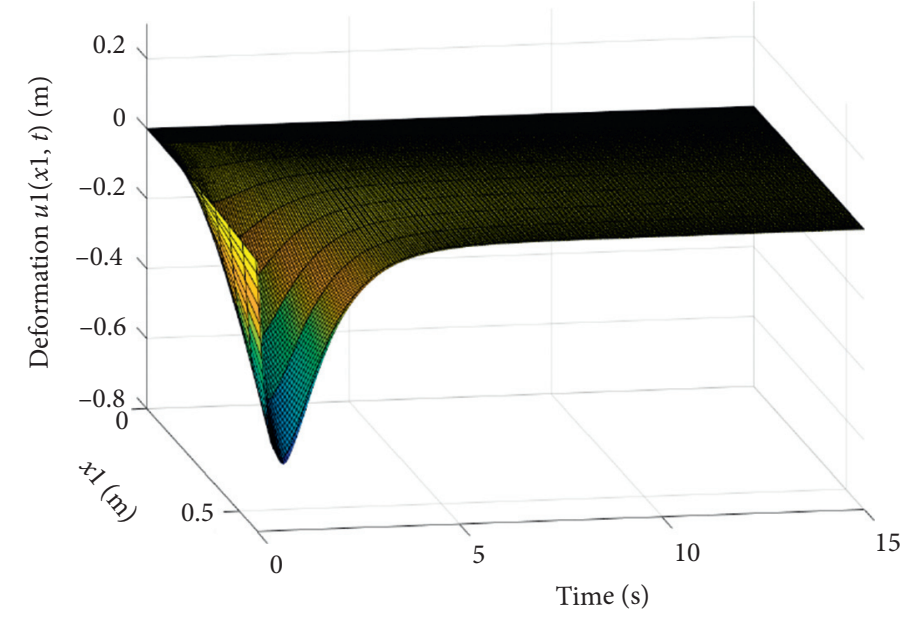

(c)

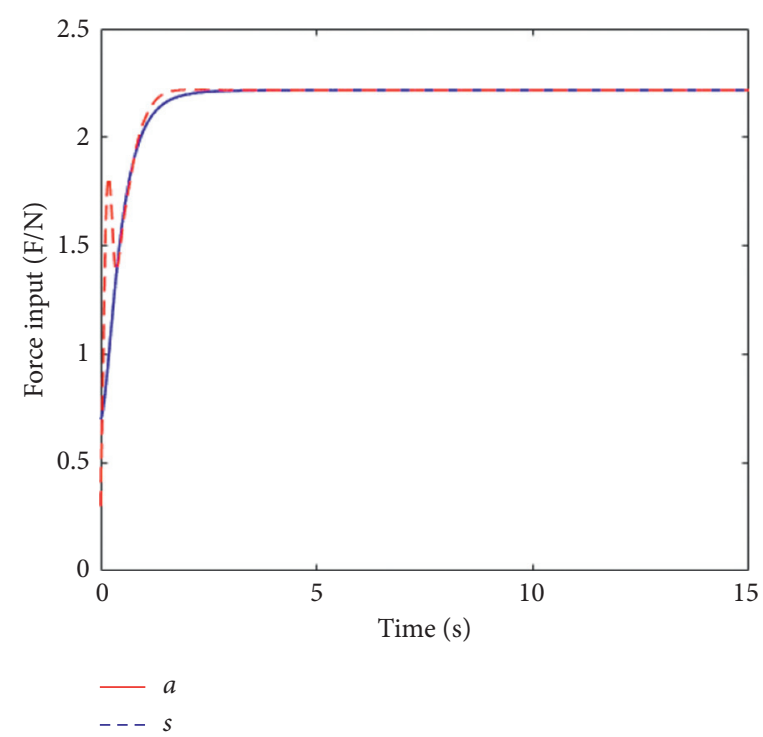

(b)

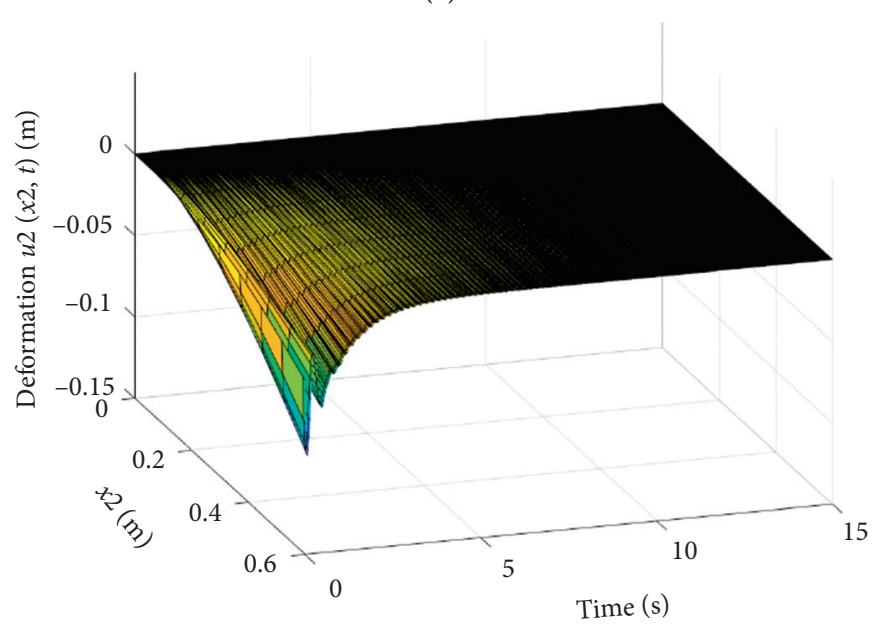

(d)

FIGURE 9: Comparison with the pure boundary control method. (a) Actual joint angles of the two links. (b) Uncertain function approach effect. (c) The deformation of link 1. (d) The deformation of link 2.

\section{Conclusions}

In this paper, a distributed parameter PDE model with ODE boundary conditions of the flexible manipulator is established first based on Hamilton's principle, in order to avoid the possible spillover instability. The boundary conditions derived from the dynamic model are further simplified.
Then, we propose an adaptive boundary control law to track the desired joint positions and suppress the vibration of flexible links simultaneously. With the combinational consideration of all uncertain parameters, namely, the joint mass and tip mass, we put forward a function to represent these parameters and design a RBF NN to approximate the parametric uncertainty. No offline training or learning 
process is needed for the proposed RBF NN, which means the approximation process is following the operation of the flexible manipulator. The extended Lyapunov direct approach is used to analyze the asymptotic stability of the combined control scheme. Simulation results illustrate that the flexible manipulator can track the desired joint angles and the vibration of flexible links is suppressed well by the proposed control law. Apart from our present work, other following aspects could be extended in further studies. RBF $\mathrm{NN}$ has its own drawbacks, such as relying on the initial values or overfitting, causing fluctuations of the control inputs. Due to the sources of uncertainty, distributed parameters are other factors needed to be considered in adaptive control area.

\section{Data Availability}

The data used to support the findings of the study can be available from the corresponding author upon request.

\section{Conflicts of Interest}

The authors declare that there are no conflicts of interest regarding the publication of this paper.

\section{Acknowledgments}

This work was supported by National Natural Science Foundation of China, no. 51775270, and a project funded by the Priority Academic Program Development of Jiangsu Higher Education Institutions.

\section{References}

[1] O. A. Garcia-Perez, G. Silva-Navarro, and J. F. Peza-Solis, "Flexible-link robots with combined trajectory tracking and vibration control," Applied Mathematical Modelling, vol. 70, no. 1, pp. 285-298, 2019.

[2] M. Shao, Y. Huang, and V. V. Silberschmidt, "Intelligent manipulator with flexible link and joint: modeling and vibration control," Shock and Vibration, vol. 2020, pp. 1-15, 2020.

[3] J. Jiang, M. Seaid, M. S. Mohamed et al., "Inverse algorithm for real-time road roughness estimation for autonomous vehicles," Archive of Applied Mechanics, vol. 90, no. 6, pp. 13331348, 2020.

[4] J. Jiang, M. S. Mohamed, M. Seaid, and H. Li, "Identifying the wavenumber for the inverse Helmholtz problem using an enriched finite element formulation," Computer Methods in Applied Mechanics and Engineering, vol. 340, pp. 615-629, 2018.

[5] T. D. Nguyen and O. Egeland, "Infinite dimensional observer for a flexible robot arm with a tip load," Asian Journal of Control, vol. 10, no. 4, pp. 456-461, 2008.

[6] J. Liu and L. Zhang, "Adaptive boundary control for flexible two-link manipulator based on partial differential equation dynamic model," IET Control Theory \& Applications, vol. 7, no. 1, pp. 43-51, 2013.

[7] W. He, X. He, M. Zou et al., "PDE model-based boundary control design for a flexible robotic manipulator with input backlash," IEEE Transactions on Control Systems Technology, vol. 27, pp. 790-797, 2018.
[8] T. Jiang, J. Liu, and W. He, “Adaptive boundary control for a flexible manipulator with state constraints using a barrier Lyapunov function," Journal of Dynamic Systems Measurement and Control, vol. 140, no. 8, Article ID 081018, 2018.

[9] R. Kumar and S. Srivastava, "Externally Recurrent NN based identification of dynamic systems using Lyapunov stability analysis," ISA Transactions, vol. 98, pp. 292-308, 2019.

[10] J. Jiang, S. Cao, and Y. Dai, "Research on RBF NN model compensation and adaptive control of robot manipulators," in Proceedings of the Chinese Control and Decision Conference (CCDC), Yinchuan, China, 2016.

[11] V. Helon, A. Hultmann, and C. Leandro, "Cascaded evolutionary algorithm for nonlinear system identification based on correlation functions and radial basis functions NNs," $\mathrm{Me}$ chanical Systems and Signal Processing, vol. 68-69, pp. 378393, 2016.

[12] X. Shi, Y. Cheng, C. Yin et al., "Design of adaptive backstepping dynamic surface control method with RBF NN for uncertain nonlinear system," Neurocomputing, vol. 330, no. 2 , pp. 490-503, 2018.

[13] A. Farmanbordar and S. M. Hoseini, "NN adaptive output feedback control of flexible link manipulators," Journal of Dynamic Systems, Measurement, and Control, vol. 135, no. 2, Article ID 021009, 2012.

[14] Z.-C. Qiu and W.-Z. Zhang, "Trajectory planning and diagonal recurrent neural network vibration control of a flexible manipulator using structural light sensor," Mechanical Systems and Signal Processing, vol. 132, pp. 563-594, 2019.

[15] Z.-H. Jiang, "Workspace Trajectory Control of Flexible Robot Manipulators Using NN and Visual Sensor Feedback," in Proceedings of the Canadian Conference on Electrical and Computer Engineering, Halifax, Canada, 2015.

[16] Y. Ding, X. Chen, M. Zhang, and J. Rong, “Anti-Windup for Two-Link Flexible Arms with Actuator Saturation Using $\mathrm{NN}$," in Proceedings of the 2010 International Conference on E-Product E-Service and E-Entertainment, pp. 1-4, Henan, China, 2010.

[17] H. A. Talebi, R. V. Patel, and H. Asmer, "NN based dynamic modeling of flexible-link manipulators with application to the SSRMS," Journal of Field Robotics, vol. 17, no. 7, pp. 385-401, 2015.

[18] J. O. Pedro and T. Tshabalala, "Hybrid NNMPC/PID Control of a Two-Link Flexible Manipulator with Actuator Dynamics," in Proceedings of the 10th Asian Control Conference, Kota Kinabalu, Malaysia, 2015.

[19] H. J. Yang and M. Tan, "Sliding mode control for flexible-link manipulators based on adaptive NNs," International Journal of Automation and Computing, vol. 15, no. 2, pp. 1-10, 2018.

[20] B. Xu, "Composite learning control of flexible-link manipulator using NN and DOB," IEEE Transactions on Systems, Man, and Cybernetics: Systems, vol. 2017, pp. 1979-1985, 2017.

[21] D. S. Broomhead and D. Lowe, "Multivariable functional interpolation and adaptive networks," Complex Systems, vol. 2, no. 3, pp. 321-355, 1988.

[22] M. Sasaki, H. Murasawa, and S. Ito, "Control of a two-link flexible manipulator using NNs," in Proceedings of the International Workshop \& Conference on Photonics \& Nanotechnology. International Society for Optics and Photonics, New York, NY, USA, 2008.

[23] C. A. Rogers, A. J. Kassab, E. A. Divo, Z. Ostrowski, and R. A. Bialecki, "An inverse POD-RBF network approach to parameter estimation in mechanics," Inverse Problems in Science and Engineering, vol. 20, no. 5, pp. 749-767, 2012. 
[24] W. He, Y. Ouyang, and J. Hong, "Vibration control of a flexible robotic manipulator in the presence of input deadzone," IEEE Transactions on Industrial Informatics, vol. 99, p. 1,2016 .

[25] C. D. Rahn, Mechatronic Control of Distributed Noise and Vibration: A Lyapunov Approach, Springer-Verlag, New York, NY, USA, 2001. 